\title{
Confirming routine stentograms after cystectomy is unnecessary
}

\author{
Naji Touma, MD; Jeffrey Spodek, MD; James Kuan, MD; Robert R. Shepherd, MD; W. Philip Hayman, MD; \\ Joseph L. Chin, MD
}

\begin{abstract}
Objective: In the current trend of earlier discharge from hospital and minimizing costs with selective intervention instead of routine "standard" orders, most institutions have abolished routine radiographic imaging of the collecting system before stent removal in postcystectomy patients, although clear supportive data from the recent literature is scarce. We retrospectively reviewed our experience with routine postoperative stentograms in 100 cystectomies to confirm that our decision to omit the stentogram procedure does not compromise patient safety and well-being.
\end{abstract}

Methods: We retrospectively reviewed the records of 100 patients who underwent radical cystectomy for bladder cancer. All 100 patients (87 with ileal conduit and 13 with orthotopic neobladder reconstruction) had their ureteroenteric anastomoses stented with feeding tubes that were exteriorized. Retrograde stentogram was performed under fluoroscopic monitoring on postoperative days 7 to 9 . The radiology reports were reviewed for any mention of extravasation.

Results: Extravasation was detected on the stentograms of 5 of 197 (2.5\%) ureteroenteric anastomoses. Clinical signs suggestive of an anastomotic leak had preceded the imaging studies in 4 of the 5 cases. After expectant management, only 1 patient $(1.0 \%)$ required intervention with percutaneous nephrostomy.

Conclusions: The relatively low extravasation rate and the infrequent need for active intervention coupled with the fact that an anastomotic leak can usually be diagnosed on clinical grounds confirms that routine use of stentogram after ureteroenteric anastomoses is not justified. Our decision to perform postoperative stentograms selectively is appropriate.

CUAJ 2007;1(2):103-5

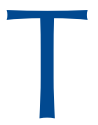
he routine use of ureteral stents after urinary diversion has reduced postoperative complications such as extravasation, fistula formation and anastomotic strictures. ${ }^{1,2}$ Management practices relating to postoperative stents have varied, ranging from routine removal at a predetermined postoperative date without radiological studies, to radiological studies performed when clinically indicated, to routine stentograms on all patients before stent removal. Published data to support the various practices have been relatively scarce. However, in the current trend of earlier discharge from hospital and minimizing costs with selective intervention instead of routine "standard" orders, most institutions have abolished routine radiographic imaging of the collecting system before stent removal in postcystectomy patients. To confirm that the decision to omit the procedure does not compromise patient safety and well-being, we retrospectively reviewed the records of 100 consecutive patients who had undergone radical cystectomy and urinary diversion when routine stentogram was still being performed at 7 to 10 days postoperative.

\section{Materials and Methods}

We reviewed the records of 100 patients (75 men, 25 women; mean age 65.4 yr) who underwent radical cystectomy for bladder cancer and who had routine postoperative stentograms before stent removal. Eightyseven patients underwent ileal conduit diversion and 13 received an orthotopic neobladder (Studer) reconstruction. Side-to-side stapled bowel anastomosis was performed. A single-layered, nontunnelled ureteroenteric anastomosis was performed with 5-O monofilament absorbable sutures. Silicone feeding tubes (5- or 8-French, depending on ureteral caliber) were used as ureteral stents across the ureteroenteric anastomoses. The distal end of the feeding tube was exteriorized via the urinary stoma in the case of ileal conduits and via side holes on the proximal ileal segment in the case of Studer neobladders. On postoperative day 7 , stentogram was performed with retrograde contrast injection via the stents under fluoroscopic monitoring on all patients. If no extravasation was demonstrated, the stents were removed. If the stentogram was unsuccessful due to dislodgement or migration or to blockage of the stent in the ileal conduits, a "loopogram" was performed instead. Accordingly, 10 patients underwent a loopogram. Our recommended routine was to administer a single dose of parenteral antibiotic (aminoglycoside or cephalosporin) just before the procedure. 
Touma et al

\section{Results}

A total of 197 stentograms were performed on 100 patients; 3 patients had a solitary kidney. In 10 patients, stentogram could not be performed (unilaterally in 8 patients and bilaterally in 2 patients, for a total of 12 of 197 [6.1\%] renal units), owing to stent migration into the ileal conduit. The subsequent loopogram on these cases showed no extravasation at the anastomotic site. Five other patients had demonstrable extravasation at the ureteroileal anastomosis unilaterally (3 right-sided, 2 left-sided) (5\% of patients, or $2.5 \%$ of the renal units). Four of the leaks occurred in ileal conduit patients and 1 in an orthotopic neobladder patient.

The stents were removed in patients who had no demonstrable leak on imaging. The patients with urinary extravasation were initially managed expectantly. Repeat imaging 3 to 6 days later on 3 patients (ureterogram in 2, intravenous pyelogram in 1) showed no further extravasation. One leak was observed clinically; with negligible Jackson-Pratt drainage after 4 days, the stent was removed with no further sequelae. The only patient who required intervention showed a persistent leak on serial ureterograms, necessitating percutaneous nephrostomy insertion with subsequent resolution of the urinary leak. Four of the 5 patients with radiographic extravasation exhibited clinical signs suggestive of an anastomotic leak that predated the imaging study (that is, persistently elevated or increasing Jackson-Pratt drainage of fluid with biochemical characteristics consistent with urinary content). There were no reported complications from the procedure, such as urosepsis or pyelonephritis.

\section{Discussion}

Stenting the ureteroenteric anastomosis after urinary diversion has been widely practised, because stenting has been shown to improve surgical outcome by reducing the incidence of fistula formation, extravasation and anastomotic strictures. ${ }^{1,2}$ Nevertheless, there is relatively little data on perioperative ureteral stent management. Practices have been variable, ranging from stent removal only after radiological confirmation of anastomotic integrity, to removal at a predetermined postoperative day without radiological studies, to using radiological examinations based on clinical signs and symptoms, such as persistent postoperative azotemia, sepsis and urinary leakage via the drains. ${ }^{3}$ In the era of fiscal constraints and pressure to shorten hospital stay and minimize costs, selective intervention instead of standard orders fits the mandate. Along with many other institutions, we have abolished routine radiographic imaging of the collecting system before stent removal in postcystectomy patients, resulting in shorter hospital stays. With this retrospective review, we wished to affirm that the decision does not compromise patient care for the sake of cost savings.

Dorph and colleagues ${ }^{4}$ reviewed patients who had undergone urethral Kock ileal bladder substitution and reported an incidence of ureteroileal anastomotic leak of $12 \%$ (6 patients), with only 1 patient requiring intervention by percutaneous nephrostomy. The radiological evaluation of these patients consisted of retrograde pyelography at the 10-day mark and pouch cystography at 2 weeks. In contrast, Manion and others ${ }^{5}$ evaluated patients who had undergone ileal conduit and continent diversion on postoperative days 5 to 8 and reported a leak rate of only $2.2 \%$. Pantuck and colleagues $^{6}$ reviewed patients who had had ileal conduits, catheterizable reservoirs or orthotopic bladder diversion and found an extravasation rate of only $0.98 \%$. However, this lower rate could be explained by the fact that stentograms were performed later (on average, on postoperative day 12). Moreover, the authors excluded patients diagnosed with urinary leaks via clinical and other radiographic means from their series.

Unlike the other series, where there was usually a time-range, the current cohort underwent stentogram 1 week postoperative. In this series, 4 of the 5 patients diagnosed with an anastomotic leak would have been identified as problematic based on clinical signs alone, primarily prompted by increased Jackson-Pratt drain volume. Thus, it appears that, for patients with no signs of increased Jackson-Pratt drainage, azotemia or sepsis, removing the ureteral stents on approximately postoperative day 7 without radiological confirmation of anastomic integrity is safe. Selective use of stentogram would be indicated in patients exhibiting signs suggestive of a urinary leak, to confirm the diagnosis and document the extent of extravasation. In such cases, serial stentogram provides guidance for management. The low intervention rate in the current series is consistent with findings by others, supporting the notion that most 
ureteroenteric anastomotic leaks will heal spontaneously, especially when a stent is still in situ.

Routine stentogram before stent removal in our series would have identified only 1 clinically unsuspected leak, giving a low yield rate of less than $1 \%$. The potential adverse effects of routine stentograms should also be considered when evaluating its use. In Pantuck and colleagues' series, ${ }^{6}$ complications attributable to the stentogram occurred in $17.6 \%$ of patients and ranged from minor contrast allergy, to transient fever, to pyelonephritis and sepsis. Moreover, the cost of administering routine stentograms is significant. ${ }^{6}$ They estimated a cost of $\$ 58000$ to detect a single urinary leak, ${ }^{6}$ albeit in a different health care system. Shortening the hospital stay (by not having to wait for the routine stentogram on postoperative day 7) contributes to the cost savings. Based on our findings, it appears that some cost savings can be achieved without compromising patient care.

\section{Conclusions}

Clinical signs and symptoms are usually adequate for the diagnosis of the relatively rare postoperative complication of uretero entericanastomotic leak after cystectomy. Routine postoperative stentogram has a low yield and appears superfluous. Selective use of stentograms based on clinical judgement still has a role.

Division of Urology, London Health Sciences Centre, University of Western Ontario, London, Ont.

This article has been peer reviewed.

Competing interests: None declared.

\section{References}

1. Jarowenko MV, Bennett AH. Use of single J urinary diversion stents in intestinal urinary diversion. Urology 1983;22:369-70.

2. Regan JB, Barrett DM. Stented versus nonstented ureteroileal anastomoses: is there a difference with regard to leak and stricture? I Urol 1985;134:1101-3.

3. Hensle TW, Bredin HC, Dretler SP. Diagnosis and treatment of a urinary leak after ureteroileal conduit for diversion. J Urol 1976;116:29-31.

4. Dorph S, Steven K, Mygind T, et al. Radiographic evaluation of the urethral Kock ileal bladder substitute. Acta Radiol 1993;34:133-8.

5. Manion SP, Waters WB, Flanigan RC. Efficacy of retrograde stentograms following cystectomy and diversion. J Urol 1997;158:776-7.

6. Pantuck AJ, Weiss RE, Cummings KB. Routine stentograms are not necessary before stent removal following radical cystectomy. J Urol 1997;158:772-5.

Correspondence: Dr. Joseph L. Chin, Suite 3250, 800 Commissioners Rd. E., London ON N6A 4G5; fax 519 685-8455; Joseph.Chin@lhsc.on.ca

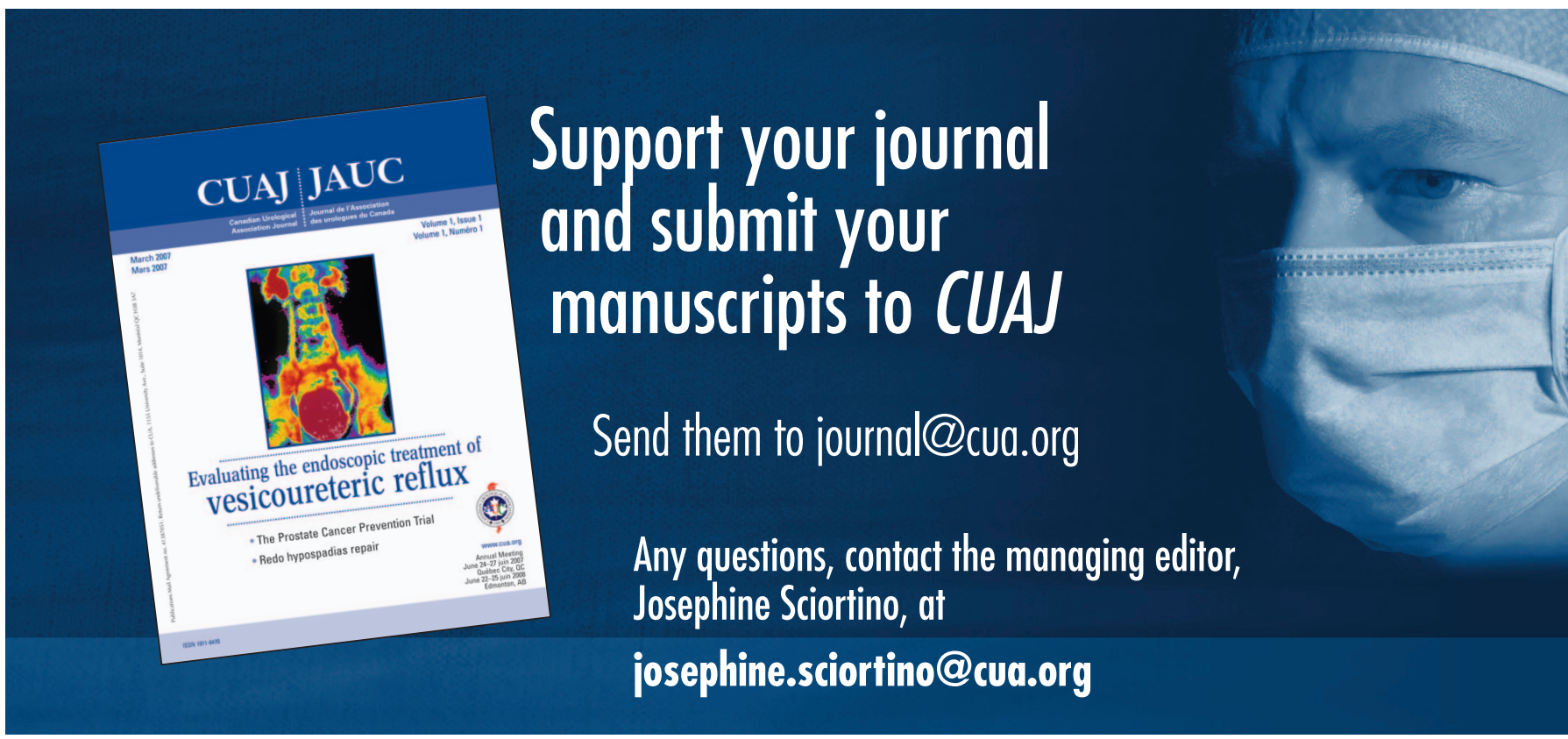

